\title{
Fünf Syrphiden (Dipt.) aus den Alpen und Karpathen.
}

\author{
Von L. Oldenberg in Berlin. \\ (Mit 4 Abbildungen.)
}

1. Chilosia atriseta n. sp. $q$. Neue Untergattung Taeniochilosia.

An einem Bergabhang des Jungbrunntals bei dessen Einmündung in das Tschamintal (etwa $1400 \mathrm{~m}$ hoch in den Tiroler D olomiten) fand ich am 27. Juni 1914 ein 우 dieser neuen Chilosia, die durch eigenartige Bildung der Fühlerborste von den bisher bekannten Arten erheblich abweicht.

Das Tier ist schwarz mit grauweifer, im ganzen kurzer Behaarung; rot ist nur das dritte Fühlerglied. Die Fühlerborste ist zu

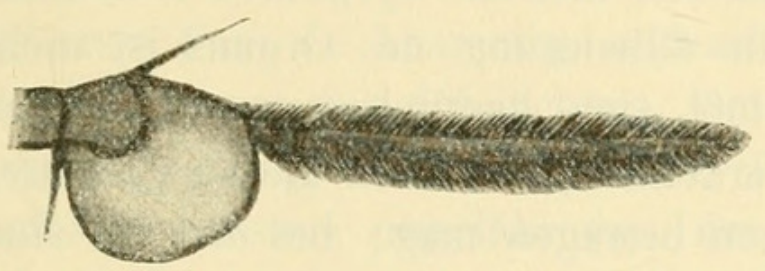

Fig. 1. Innenseite eines Fühlers von Chilosia atriseta n. sp.

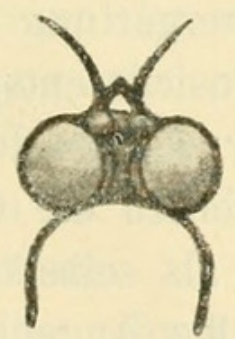

Fig. 2. Kopf von oben.

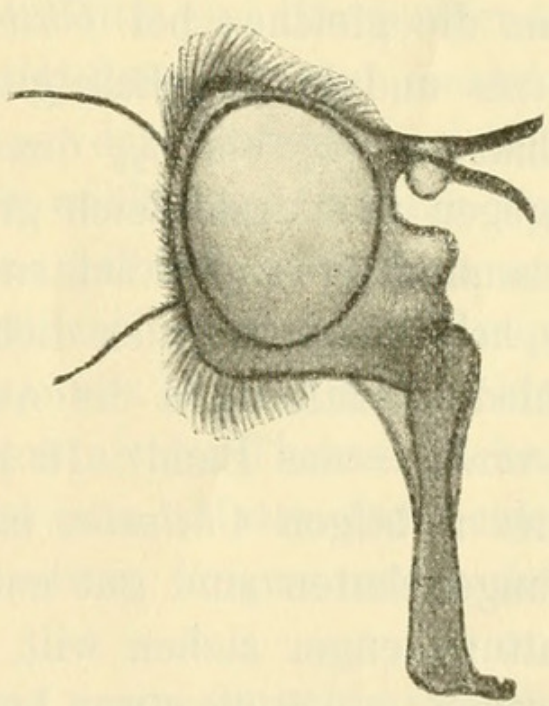

Fig. 3. Kopf von der Seite.

einem bandförmigen Griffel verbreitert, mit dichter, schwarzer Fiederung. Augen kahl; Gesicht unbehaart, mit kurz halbkugelig vorspringendem Höcker und reichlich ebensoweit vortretendem unterem Mundrand. Schildchen sechsborstig. Flügel gebräunt. Körperlänge: gut $8 \mathrm{~mm}$, ohne Fühler.

Grundfarbe glänzend schwarz, am Thorax und Hinterleib nur wenig zu Blaugrün neigend; der Glanz ist durch Behaarung und 
Bestäubung nicht sehr beeinträchtigt (am Kopf und Thorax etwas, am Gesicht überhaupt nicht). Augen so gut wie kahl (nur mikroskopisch äußerst kurz weitläufig behaart). Fühler (Fig. 1) mit der „Borste" reichlich von der Stirnlänge. Die beiden ersten Fühlerglieder schwarz; erstes becherförmig, etwas kleiner als das zweite, am Ende rings mit einem Kranz weißer Härchen; zweites mit einem senkrechten Kranz feiner schwarzer Borsten, von denen die untersten und eine oberste sich durch Größe auszeichnen; es greift innen mit einer kleinen, flachen Erweiterung auf das dritte über. Letzteres ist für eine Chilosia verhältnismäßig klein, matt hell gelbrot, nur oben am Grunde ein wenig dunkler, querrundlich, vorn ein wenig eckiger begrenzt. Fühlerborste tiefschwarz, stark verdickt und seitlich abgeplattet, daher einen bandförmigen Griffel bildend, mit kurz verschmälertem Ende, etwa $2 \frac{1}{2}$ mal so lang wie das dritte Fühlerglied, mit dicken, scharfspitzigen, auf der Ober- und Unterseite län. geren Fiedern dicht und gleichmäßig besetzt. Die Gestalt der eigentlichen Fühlerborste (aus verdicktem Grunde lang haarförmig zulaufend, eventuell mit nach dem Ende hin abnehmender Härchenbekleidung), wie sie Chilosia und den meisten Syrphiden zukommt, ist also hier aufgegeben. Auch die Gliederung des Organs ist nicht ganz die gleiche: bei Chilosia läßt sich deutlich nur ein äußerst kurzes und kleines Basalglied ${ }^{1}$ ) erkennen, das nach ganz ungefährer Schätzung $1 / 15$ bis $1 / 20$ des Ganzen betragen mag; bei atriseta sind dagegen zwei fast gleich große Basalglieder deutlich entwickelt, die zusammen etwa den achten Teil der Griffellänge ausmachen mögen; sie heben sich aus der dichten Fiederung wenig ab. Diese Unterschiede rechtfertigen die Aufstellung der Untergattung Taeniochilosia ( $\dot{\gamma}_{i} \tau$ :vi $\alpha=$ das Band). In jeder anderen Hinsicht entspricht die Art einer richtigen Chilosia, namentlich in der Kopfbildung; auch die Wangenplatten sind gut entwickelt. Wer freilich die Grenzen einer Gattung enger ziehen will, könnte $T$. auch als selbständige Gattung ansehen. - Stirn etwas breiter als eine halbe Augenbreite, seitlich mit je einer deutlichen, schmalen Längsrinne, die neben den oberen zwei Dritteln des Augenrandes bis zu der recht schwachen Querfurche reicht; über letztere erstreckt sich eine noch flachere, breite Längs-

1) Da sich bei manchen Syrphiden-Gattungen deutlich eine Dreigliedrigkeit der Fühlerborste oder des Fühlergriffels wabrnehmen läßt (z. B. Pelecocera, Lampetia), ist jenes Basalglied bei Chilosia vielleicht als zweites anzusehen, und es könnte, tief eingesenkt in das dritte Fühlerglied, noch ein erstes Borstenglied oder das Rudiment eines solchen vorhanden sein; ebenso bei manchen anderen Syrphidengattungen. 
einsenkung. Die Stirn ist mit aufrechten, schwarzen Härchen bedeckt, nur zwischen Augenrand und Seitenfurche stehen grauweiße, mehr abwärts gerichtete Härchen; am Stirndreieck wird die Behaarung länger, der hintere Augenrand trägt oben ziemlich lange schwarze Haarborsten (mindestens so lang wie die halbe Stirnbreite). Behaarung des Hinterkopfes grauweiß, oben kurz, unten länger, bis doppelt so lang. Wangen bandförmig, etwa so breit wie der gefiederte Fühlergriffel, mit kurzen, feinen, weißlichen, nach unten hin länger werdenden Haaren. Gesicht glänzend schwarz, kahl (nur ganz unten Spuren von Bereifung und winzige, graue bis schwarze Härchen). Gesichtsform etwa mit der von crassiseta Lw. vergleichbar. Gesicht unter den Fühlern merklich ausgehöhlt; der Gesichtshöcker ist nicht ganz so schmal, mehr halbkugelig; der unterste Gesichtsteil reichlich ebenso weit vorgezogen wie der Höcker und am Ende etwas herabgebogen (Fig. 2 und 3).

Thorax mit verhältnismäßig kurzer, grauweißer Behaarung, die an manchen Stellen von weitläufig stehenden, dunkleren Haaren überragt wird. Schildchen von der gleichen Beschaffenheit, am Rande mit drei Paar dünner Borsten, die nach hinten an Größe zunehmen: das erste Paar ist haarartig, schwach, das letzte, größte, nicht ganz von der Länge des Schildchens. Hinter der Mitte eine durchgehende, bogenförmige Querfurche. Auf dem Postalarkallus je 2 von einander entfernte Borsten, ungefähr von der Länge der hintersten Schildborsten. Brustseiten nur wenig bereift, mit weißlicher, lockerer, längerer Behaarung. Schüppchen weif mit etwas gelblichen Rändern und weiler Randbehaarung. Schwinger mattgelb mit gebräuntem Knopf.

Hinterleib an den vorderen Tergiten etwas länger, an den hinteren ebenso lang oder etwas kürzer behaart als der Thorax; Behaarung abstehend, von der gleichen blassen Farbe. Unter den beiden ersten Sterniten ist die dünne Behaarung etwas länger, unter dem vierten sehr gering.

Beine völlig schwarz mit sehr kurzer, grauweiß schimmernder Behaarung; nur an den Schenkeln ist diese erheblicher, namentlich auf der Rückseite der vorderen Schenkel. Vordere Fersen schmal und viel kürzer als die zugehörigen Tarsensummen. Hinterschienen etwas gebogen, in der Basalhälfte gegen die Mitte hin innen mit einer sanften Schwellung; Hinterfersen schwach verdickt, etwa gleich $2 / 3$ der Schienen und gleich der Tarsensumme.

Flügel ziemlich lang, lebhaft schwarzbraun getrübt, hauptsächlich an der Wurzel (vor den Basalzellen) und der Spitzenhälfte; das braune Randmal ist mäßig dunkel. Adern ohne besondere auffällige 
Verstärkungen; hintere Querader nur wenig dicker als die benachbarten Adern. Spitzenquerader nicht besonders lang, unten mit Anhang, nur sanft geschwungen, ziemlich spitzwinklig in die fast gerade verlaufende Cubitalis mündend. Auch die Radialis ist sehr gestreckt, am Ende nur wenig geschwungen. Endbegrenzung der zweiten Hinterrandzelle gerade.

\section{Chilosia claviventris Strobl @. (Fig. 4.)}

Strobl hat das o nach einem Exemplar beschrieben (Dipt. v. Steiermark, II. Nachtr. 1909, S. 104); ich fand 4 o, die ich für diese Art halten muf, im Juli und August in Tirol

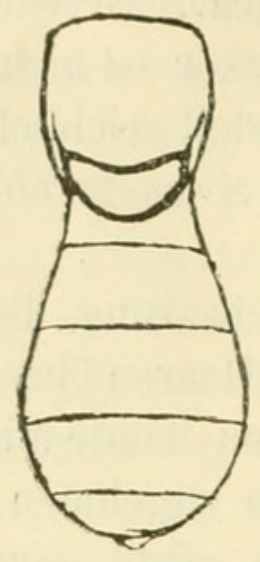

Fig. 4. (3 zwischen Trafoi und dem Stilfser Joch, 1 bei Campiglio). Jene Beschreibung paßt im ganzen auch auf das $O$; es sind folgende Abweichungen und Zusätze zu vermerken.

Die ziemlich lebhafte Färbung von Kopf und Thorax schwankt bei den verschiedenen Exemplaren zwischen grün, kupferig und violett. Stirnbreite etwas größer als die halbe Breite eines Auges. Die Längsfurchen der Stirn sind fast erloschen, die Querfurchung ist deutlich und stark. Auf dem unteren Stirnteil macht sich zuweilen ein Anflug von Bereifung bemerkbar. Ozellenhöcker stark vortretend; Haarborsten am oberen Augenrand ungefähr so lang wie die seitlichen Haare der oberen Stirn. Der kleine Gesichtshöcker ist ungefähr so schmal wie bei crassiseta Löw. Fühlerborste kurz, nur von Fühlerlänge. Der Thorax (nebst Schildchen) erscheint durch ziemlich grobe Punktierung etwas matter als der Kopf und Hinterleib. Die sonst kurze Behaarung des Schildchens wird hinten ein wenig länger, fast borstenartig, ohne daß sich jedoch einzelne auffallende Borsten herausheben. Schwinger mattgelb (nicht braun), auch der Knopf. Schüppchen weißlich mit hellem Randflaum. Hinterleib recht flach, aus schmaler Basis keulenförmig verbreitert, zuletzt wieder verschmälert; am breitesten etwa am Ende des dritten Ringes. Der Hinterleib ist grünschwarz, glatt, überall stark glänzend (nicht in der Nitte matt wie beim $\sigma^{7}$ ), mit sehr feiner, schwacher, weißlicher Behaarung, die an den hinteren Ringen oben wie unten sehr kurz, an den Seiten der beiden vordersten Tergite länger und dichter ist; auch das Hinterleibsende ist oben und unten etwas länger behaart. Beine ganz schwarz, ziemlich kahl. Der verhältnismäßig breiten Flügelform entsprechend ist die Spitzenquerader stark S-förmig ge- 
rundet, ihr oberes Ende steht ungefähr senkrecht auf der Diskoidalis oder ist gar rücklaufend gerichtet, wie bei manchen Orthoneuren; unten findet sich ein kurzer Aderanhang (auf dem linken Flügel eines Stückes auch oben). Die hintere Querader liegt der Basis der Diskoidalzelle so nahe, daß sie von deren Oberrand viel weniger als $1 / 3$ abschneidet.

3. Didea alneti Fall. var.

Am 1. August 1914 fand ich bei San Martino di Castrozza in Tirol ein Didea-Q, das als Hochgebirgsvarietät von alneti gelten muß trotz verschiedener Abweichungen, hauptsächlich in der Hinterleibszeichnung.

Die 3 hellgrünen Fleckenpaare des Hinterleibes sind sämtlich viel schmäler und unter sich gleichartiger als sonst; sie hängen nirgends zusammen, sondern sind alle breit unterbrochen. Die Flecken des 1. Paares halten innen den normalen Abstand voneinander, sind aber viel weniger breit gerundet, daher schmäler und parallelrandiger. Die innen spitz zulaufenden Flecken des 2. und 3. Paares liegen fast wagrecht, indem die Unterränder eines jeden Paares nur wenig divergieren, und sind auffallend schmal (ihre Aufenenden nehmen weniger als die Hälfte des seitlichen Tergitrandes ein, bei meinen Thüringer Normalexemplaren reichlich die Hälfte). Die Mittelflecken sind innen nicht ganz so weit, die hintersten Flecken erheblich weiter voneinander entfernt als die vordersten. Die geschwärzten Zeichnungen des Kopfes sind ein wenig ausgedehnter, ebenso die Schwärzungen der Beine. Das Schildchen ist auf der Vorderhälfte blaß und schwach behaart (sonst gleichmäßiger und ziemlich dunkel behaart). Die Einkerbung der Cubitalader ist ungefähr ebenso tief, jedoch viel spitzwinkliger.

\section{Brachyopa arcuata Panz. var. lateralis $\mathrm{m}$.}

Ein $\sigma^{7}$, am 5. Juni 1904 bei Herkulesbad gefangen. Von der Hauptform der arcuata durch rotgelbe Einfassung des Thoraxrückens, besonders an den Seiten, und durch fehlende Flügelpunkte verschieden.

Am Thorax sind nicht nur die Brustseiten (mit Ausnahme der untersten, über den Hüften liegenden, dunkleren Partien) rostrot, sondern auch über der Dorsopleuralnaht ein breiter Rückenstreifen, der die glänzend rotgelben Schulterschwielen mit einem schmalen Saum umfaßt und vorn zwischen ihnen und dem schwärzlichen Mittelstreif des Rückens als roter Fleck endigt; auch vor dem Schildchen liegt eine schmale rötliche Querzone. Die beiden sonst für arcuata 
charakteristischen Punkte auf jedem der schwach gelblich gefärbten, gelbbraun bis schwarzbraun geaderten Flügel fehlen völlig. An der Stelle, wo sonst der schwarze Punkt der 1 . Hinterrandzelle liegt, ist die Diskoidalader nicht so eckig vorgewölbt, sondern flacher gebogen, bei nur geringer Verstärkung der dortigen Querader. Die sogenannte Spitzenquerader ist fast gerade gestreckt. Das schnauzenförmige Gesicht erscheint ein wenig, doch nur ganz unerheblich, länger und spitzer ausgezogen als in der Regel. Da sich in allen übrigen Merkmalen plastische Übereinstimmung mit arcuata ergibt (die kleinere Art bicolor Fall. unterscheidet sich, abgesehen von den bekannten, auffälligeren Eigenschaften auch durch etwas kürzere Thoraxbehaarung und kürzere Pubeszenz der Fühlerborste), nehme ich an, daß nur eine Varietät dieser Art vorliegt, um so mehr, als ich von Thalhammer eine ungarische arcuata erhalten habe, deren Flügelpunkte beinahe fehlen, während die Adern dort fast wie bei meinem Exemplar verlaufen.

\section{Zelima (Xylota) pannonica n. sp.}

Bei Herkulesbad, wo einst Kow ar z die Zelima rufipes Löw entdeckt hat, fand ich im Juni 1904, sodann wieder im Juni und Juli 1912 sechs $\sigma^{\top}$ einer weiteren, neuen Art: pannonica m., die von den drei ähnlichen Arten femorata L., curvipes Lw. und rufipes Lw. durch kürzere Behaarung des Körpers sowie durch abweichende Färbung des Gesichtes und der Beine leicht zu unterscheiden ist.

Behaarung namentlich am Hinterkopt und Thorax erheblich kürzer; letzterer erscheint mit seiner unscheinbaren Grund- und schwachen, dünnen, über sie hinausragenden Haarbekleidung viel kahler als bei jenen Arten. Auch die Beine sind wesentlich schwächer behaart: besonders an den vorderen Schenkeln ist die gelbliche Behaarung, welche hauptsächlich an der Rückseite bei jenen Arten auffällt, lange nicht so ausgebildet.

Während bei meinen Exemplaren der drei bekannten Arten die Grundfarbe von Stirn und Gesicht glänzend schwarz ist mit rein silberweißer Bestäubung (nur bei einem femorata- $\sigma^{\top}$ in der Nähe der Fühlerbasis rötlich), geht bei pannonica die Bestäubung, oben auf der Stirn silberweiß, a uf dem Gesicht nach unten hin mehr ins Weifgelbliche über, und der aufgeworfene Mundrand is t breit rotgelb. Erstes Fühlerglied schwarzbraun, auch das zweite etwas gebräunt. Fühlerborste aus rotgelbem Grunde allmählich mehr oder weniger verdunkelt: die ganze Fühlerborste daher 
bald heller, bald dunkler. Am Hinterleib sind Spuren von Seitenflecken, die sich bei jenen Arten meist als schwarzbläulich glänzende Stellen vom matter schwarzen Grunde abheben, kaum noch wahrzunehmen. Randmal der Flügel dunkelbraun (so auch bei meinen rufipes, nicht eigentlich schwarz, wie Löw angibt). Schwinger hell rotgelb, noch heller als die Farbe der Beine (bei meinem $\sigma^{\pi}$ von curvipes ebenfalls, bei den rufipes dagegen dunkelbraun; auch bei femorata meist reichlich dunkel braun, nur zuweilen heller).

Hüften und Schenkelringe (bei allen vier Arten) dunkel. Durch die Beinfärbung weicht pannonica am meisten ab. Die Schenkel der vorderen Beine, namentlich der Mittelbeine, sind bei meinen Exemplaren am Grunde in geringerer oder größerer Ausdehnung gebräunt, schwächer oder stärker (nur bei einem Stück Vorderschenkel ganz rot; bei meinen Exemplaren der anderen Arten vordere Schenkel ganz rotgelb); von den Tarsen dieser Beine sind mindestens das erste und zweite Glied fast ganz rotgelb (mit Ausnahme der äußersten Spitzen), die beiden Endglieder dunkelbraun. An den Hinterbeinen der drei anderen Arten ist immer ein wesentliches, kürzeres oder längeres Endstück des Schenkels ringsum tiefschwarz gefärbt mit scharfer vorderer Begrenzung, der Rest des Beines ganz schwarz; bei pannonica dagegen sind die Hinterschenkel rein rotgelb, nur unter der Schenkelspitze kurz vor dem Kniegelenk liegt ein kleines dunkles Fleckchen; Hinterschienen am Grunde breit rotgelb, nach dem Ende hin allmählich in Schwarzbraun (nicht Tiefschwarz) übergehend; Tarsen ziemlich schwarzbraun, zuweilen stellenweise gerötet. Dicke der Hinterschenkel und Krümmung der Hinterschienen ungefähr wie bei femorata.

Größe: wie rufipes. - Von letzterer fing ich bei Herkulesbad gleichzeitig zwei Pärchen, während mir femorata und curvipes dort nicht begegnet sind. 


\section{$2 \mathrm{BHL}$ Biodiversity Heritage Library}

Oldenberg, Lorenz. 1916. "Fünf Syrphiden (Dipt.) aus den Alpen und Karpathen." Wiener entomologische Zeitung 35, 101-107. https://doi.org/10.5962/bhl.part.9986.

View This Item Online: $\underline{\text { https://www.biodiversitylibrary.org/item/45303 }}$

DOI: https://doi.org/10.5962/bhl.part.9986

Permalink: https://www.biodiversitylibrary.org/partpdf/9986

\section{Holding Institution}

Smithsonian Libraries

\section{Sponsored by}

Smithsonian

\section{Copyright \& Reuse}

Copyright Status: NOT_IN_COPYRIGHT

This document was created from content at the Biodiversity Heritage Library, the world's largest open access digital library for biodiversity literature and archives. Visit BHL at https://www.biodiversitylibrary.org. 